\title{
Evaluation of a circuit training program for people with Parkinson's disease: a pilot study
}

\begin{abstract}
Objective: The 3 objectives of this study were to determine if a circuit group exercise program targeting balance was effective and feasible for patients with Parkinson's disease; to determine if any improvements were sustained at 6 months post-intervention and to evaluate patients' quality of life pre- and post-intervention.
\end{abstract}

Design: A pre and post observational clinical study.

Subjects: Participants were community-dwelling patients with PD referred outpatient rehabilitation at metropolitan hospital.

Methods: The intervention involved a weekly 1-hour circuit training class conducted for 8 weeks. Clinical measures of balance, mobility and quality of life were taken on commencement of the program, on completion of the program and at six months post completion.

Results: There were significant improvements in some aspects of balance following completion of the program however these were not sustained at 6-months postintervention. Self-rated health status was generally poor, but program satisfaction was high.

Conclusion: For this group of patient circuit balance classes were feasible and safe. Further studies are required to better examine the effects of this treatment approach for people with Parkinson's disease. The 3 objectives were to determine if a groupbased exercise program targeting balance was effective in patients with Parkinson's disease (PD); determine the sustainability of any improvements at 6 months postintervention and evaluate quality of life (QoL) pre- and post-intervention. Participants are community-dwelling patients with PD and balance impairment attending circuit balance classes between 2007 and $2010(\mathrm{~N}=19)$. The intervention involves a weekly 1-hour group-based circuit training exercise class over 8 weeks. The outcome measures were: Timed Up and Go, Functional Reach, Step Test, and EQ-5D Health Questionnaire etc. There was a significant improvement in performance on the Step Test after the intervention. This was not sustained at 6-months post-intervention. Selfrated health status was generally poor, but program satisfaction was high. Circuit balance classes result in a short-term improvement in balance in patients with PD, but do not appear to alter QoL. The exercise intervention may offer additional benefits.
Volume 2 Issue I - 2018

Ling Lan,' Leah Goodwin, ${ }^{2}$ Paul Bew ${ }^{3}$

'Geriatric and Rehabilitation Medicine, The Prince Charles Hospital, Australia

${ }^{2}$ Rehabilitation Medicine, Gold Coast Hospital, Australia

${ }^{3}$ Brighton Rehabilitation Unit, Brighton Health Campus, Australia

Correspondence: Ling Lan, Geriatric and Rehabilitation Medicine, The Prince Charles Hospital, Rode Road, Chermside, Queensland,Australia, Email ling.lan@health.qld.gov.au

Received: December 13,2017 | Published: January 24, 2018

Keywords: parkinson's disease, circuit training, balance, outpatient rehabilitation

Abbreviations: PD, parkinson's disease; NICE, national institute for health and clinical excellence; QoL, quality of life

\section{Introduction}

Parkinson's disease (PD) is a chronic, degenerative and progressive disorder of movement. It results in a high burden of disease for the individual $^{1}$ and carries with it a significant economic burden and social cost. ${ }^{1,2}$ This raises concern about the future management of PD, particularly given that Tthe prevalence of the disease is predicted to increase by $85 \%$ by 2025 due to the ageing population. ${ }^{2}$.

The motor features of PD include, presented as tremor, rigidity and brady kinesia. This predispose the patient to gait impairment, postural instability and falls. ${ }^{3,4}$ There are also a range of non-motor features of $\mathrm{PD}$, including autonomic dysfunction, neuropsychiatric disturbance and cognitive impairment, which may further contribute to the risk of falling. ${ }^{4}$ Falls and fractures have been well documented to cause significant morbidity ${ }^{5}$ and mortality ${ }^{6}$ in older people and have been shown to be the most common reason for patients with PD to require admission to hospital. ${ }^{7}$ Poor Imbalance is also an independent risk factor for admission to a nursing home for patients with PD. ${ }^{8}$

UPrevious research has shown that up to $60 \%$ of people with PD report functional limitation in walking ability, ${ }^{1}$ Gwith gait impairments includeing reduced gait velocity, stride length and a shuffling patternfoot clearance. ${ }^{9}$ Impairments of postural control in people with PD are complex., Possible reasons for this includewith the aetiology proposed to include reduced inter-limb coordination; reduced proprioception somatosensation; asymmetry in gait and balance control; difficulties initiating compensatory movements; and axial stiffness. ${ }^{4}$ The most challenging balance tasks for patients are turning, standing with a narrow base of support and standing on one foot. ${ }^{10}$ Patients with PD have also been shown to overestimate their limits of stability, an effect which is compounded with worsening disease. ${ }^{11}$ Pharmacological therapy with levodopa only provides temporary relief of symptoms and its efficacy decreases over the duration of its use. ${ }^{7-9,12}$ Therefore as such, it is important that nonpharmacologic interventions such as ambulatory rehabilitation are fully explored. ${ }^{9}$ 
Exercise and motor training are known to improve balance and mobility in people with Parkinson 's disease ${ }^{10}$ The National Institute for Health and Clinical Excellence (NICE) in the United Kingdom has recommended that all patients with PD should have access to physiotherapy to maximize their functional status. ${ }^{11}$ There is evidence to support exercise interventions in people with $\mathrm{PD}$, with positive effects on physical functioning, health-related quality of life, strength, balance and gait speed shown. ${ }^{12}$ The best way of providing this rehabilitation is not presently known. ${ }^{8,13}$

Circuit class therapy describes a model of therapy delivery that utilises active exercises and activities that are task specific. ${ }^{14} \mathrm{~A}$ key component of circuit class therapy is that there is a focus on repetitive practice of everyday functional tasks and continual progression of exercises. ${ }^{15}$ Participants may complete a series of workstations arranged in a circuit ${ }^{15}$ or may complete a series of individualised exercises within a group setting. ${ }^{16}$ Circuit class therapy differs from the conventional one therapist to one patient model as there are usually more than two patients involved at any one time. ${ }^{16}$ Circuit class therapy has been shown to be effective in improving the mobility of people early after stroke, ${ }^{16}$ in chronic stroke patients ${ }^{17}$ and in people with a history of falls ${ }^{18}$ Circuit class therapy has the potential to be a more effective means of providing a greater amount of physical therapy for people both within the hospital setting and in the community outpatient setting. ${ }^{14}$ The efficacy of circuit class training has not been evaluated for people with Parkinson's disease.

Howe et al. ${ }^{13}$ showed that exercise results in statistically significant improvements in balance in older people generally, with the forms of exercise including gait and balance tasks, muscle strengthening, walking and functional activities. Whilst the study excluded patients with a diagnosis of $\mathrm{PD}$, it is a natural extension to consider that exercise interventions may be beneficial to this patient population also. ${ }^{14,15}$ One proposed mechanism, based on animal models, is that the symptoms of PD can be eased by exercise by increasing dopamine synthesis in residual striatal nerve cells. ${ }^{15}$

In an ambulatory rehabilitation setting, exercise interventions targeting balance are commonly offered by physiotherapists in a group environment. ${ }^{16-19}$ However, a review of the literature finds that there is a recurrent issue with the definition of what constitutes physiotherapy, as there is no uniform treatment approach. ${ }^{12,20,21} \mathrm{~A}$ wide variety of exercise interventions make it difficult to determine which component is providing benefit. ${ }^{13,21,22}$ The recent publication of evidence-based guidelines for physical therapy in PD attempts to address these issues. ${ }^{21}$

A Cochrane systematic review published in 2001 concluded that there was insufficient evidence to support or refute the efficacy of physiotherapy to treat PD. ${ }^{12}$ Despite this, the National Institute for Health and Clinical Excellence (NICE) in the United Kingdom recommended in its clinical guidelines for PD in 2006 that all patients should have access to physiotherapy to maximise their functional status. ${ }^{23} \mathrm{~A}$ more recent systematic review and meta-analysis found that there was evidence to support exercise interventions in people with $\mathrm{PD}$, with positive effects on physical functioning, health-related quality of life, strength, balance and gait speed, but not in reducing falls risk or depression. ${ }^{22}$

These conflicting study results create uncertainty about the effectiveness of exercise interventions for people with PD, particularly given the progressive nature of the condition. Further, there is a paucity of studies identified which evaluate the sustainability of any positive gains in the target population following program completion, ${ }^{13}$ nor the optimal dose or duration of treatment. ${ }^{16}$ This also raises questions about the cost-effectiveness of the programs, a key consideration when designing interventions to reduce the burden of disease and economic cost of PD. ${ }^{2,18}$

The is study aims of this study were to determine if:

i. Circuit class therapy was safe and effective in improving the balance and mobility of people with Parkinson's disease.

ii. Any improvements gained were sustained at six months post intervention and

iii. There were any changes in the patients' reported quality of life following the intervention.

To address these issues by evaluating the outcomes of the groupbased exercise program targeting balance (circuit balance class, CBC) currently provided to clients with PD at The Prince Charles Hospital (TPCH), Brisbane, as part of the routine ambulatory rehabilitation program. This evaluation study by pre and post intervention will address the following key research questions:

i. Is CBC effective in improving balance and walking performance in patients with PD?

ii. Is any improvement gained sustained at 6 months postintervention?

iii. How do patients rate their Quality of Life (QoL) before and after the intervention?

The hypothesis is that ambulatory circuit balance classes are effective in improving gait, balance and health-related QOL in the short-term, however are unlikely to provide a sustained benefit at 6 months post-completion. Whilst it does not include a costeffectiveness analysis, the research may offer an insight into the economic rationality of providing such an ambulatory program to patients with a degenerative neurological disease if benefits are not sustained. ${ }^{18}$

\section{Methods}

This study was designed as a pre and post analysis observational study and utilizing patient data collected before and after attendance at CBC. The study was approved by the Human Research and Ethics Committee of The Prince Charles Hospital.

All identified patients referred to the Outpatient Rehabilitation Day Hospital with a diagnosis of PD and who attended at least one circuit class CBC at TPCH between 2007 and 2010 were included in the study. Referrals were to TPCH ambulatory rehabilitation service is received from multiple sources, including local neurologists, general practitioners and hospital allied health staff. The sample patients commenced the circuit classes following were admitted to the $\mathrm{CBC}$ after an assessment by a multidisciplinary assessment team, including involving a physiotherapist, medical officer and nurse. Inclusion Admission criteria were required the patient had to have an identified balance and/or mobility impairment and were to be able to participate in a weekly circuit group-based exercise program. Exclusion criteria were an Patients were not admitted to the program if they had an unstable medical condition that precluded exercise, poorly managed incontinence, a requirement for constant supervision of mobility, or if they were unable to follow two-stage commands. 
Circuit balance classes were held for 1 hour per week over a period of 8 weeks. The classes were supervised by a physiotherapist and physiotherapy assistant, with a maximum staff to patient ratio of 1:5. The classes focused on lower limb strengthening, balance and endurance, in line with physiotherapy strategies recommended for patients with PD. ${ }^{21}$ Participants rotated through a total of six6 six-minute stations, individually selected by the class supervisor. The exercise each workstation was individually tailored to the patient and tasks targeted static and dynamic balance, fitness and endurance, strengthening, and walking practice, and could be graded to add degrees of difficulty (Table 1). Rest periods were incorporated between stations.

Table I Circuit balance class exercises

\begin{tabular}{ll}
\hline Exercise task & Description \\
\hline Sit to stand & Sitting to standing from a plinth \\
Standing reach & Reaching to stack cones beyond arms length in standing \\
Stepping & Stepping with one foot to visual targets on the floor - front, side \& behind \\
Kicking a ball & Two subjects between parallel bars \\
Throwing/catching & Two subjects between parallel bars - throwing and bouncing \\
Walking - sideways & Along parallel bars \\
Walking - obstacles & Over foams, balance boards, markers between parallel bars \\
Stair climbing & Repeatedly walking up and down a small set of stairs with handrail \\
Step-ups & Stepping up and down onto a block, varying height, lead foot \& direction \\
Treadmill & Continuous walking at comfortable speed with gait pattern feedback \\
Exercise bicycle & Pedalling at comfortable speed with progression of speed \& distance \\
\hline
\end{tabular}

Clinical measures of balance and mobility were assessed All patients underwent an individual assessment before commencing and immediately after upon completion of the program. A follow up assessment was conducting $\mathrm{CBC}$, where physical performance measures were conducted by a physiotherapist. A further assessment was scheduled for 6 months after discharge from the program to review progress. The outcome clinical measures of balance taken were used included Functional Reach, Timed Up and Go, Step Test, Six Minute Walk Test and the Ten Meter Walk Test. For each test, the procedure as originally described in the literature as followed. These tools have been previously validated to assess balance, , $^{13,19,20,24}$ and walking performance 25,26 and their utility has been demonstrated in patients with PD. ${ }^{10,21,23-25,27,28}$ The patient's demographics, PD history and falls history were also documented collected from patients and their medical charts. Statistical analysis was undertaken in This information was collected for all patients who met the study criteria by undertaking a retrospective chart analysis and entering the data into a Microsoft Excel spreadsheet.

A survey was mailed to all participants in order to determine their health-related Quality of life (QoL) before and on after completion of circuit program balance classes. QoL was assessed using the Euro Qol (EQ-5D) score. ${ }^{26,27}$ EQ-5D is a standardized instrument for use as a measure of health status and outcome. Using the Australian version of the EQ-5D Health Questionnaire ${ }^{29}$ was used. Participants were asked to rate their health status in five domains (mobility, personal care, usual activities, pain and anxiety/depression) and to rate their overall health state on a vertical visual analogue scale range from 100 (best imaginable health states) to 0 (worst imaginable health states).

Further, they on completion of the program, patients were asked to rate their overall satisfaction with the program on a 7-point scale, where 1 was "very dissatisfied" and 7 was "very satisfied". Consent to participate was implied by return of the enclosed questionnaire and confidentiality was maintained by de-identifying all data.

The data were expressed as mean+/-standard errors (SE). The Median (inter quartile range) was used in the data of very high fall rates and EQ-5D Index (utility score). EQ-5D utility score was calculated using the formula of reference weights from Dolan UK preference weights. ${ }^{29,30} \mathrm{EQ}-5 \mathrm{D}$ scores range from poor health outcome at -0.594 to the best health outcome at 1.000 . Statistical analyses were performed using paired two-tailed t-tests, in addition to the use of descriptive statistics.

\section{Results}

A total of 19 patients were identified who met the study inclusion admission criteria. Four patients attended for two blocks of circuit training $\mathrm{CBC}$, with data from these attendances analysed separately. One subject did not complete the block of circuit balance classes and 5 subjects did not attend for a 6 month follow-up assessment. The mean time to follow-up was 7.6 months (SE 1.73).

The mean age of subjects was 70.8 years (SE 1.86) with a, range of from 52 to 86 years. All patients were English-speaking and lived in the community-dwelling. Twelve of the 19 patients, 12(63\%) lived in low-set accommodation and $12(63 \%)$ were of male. Eleven gender and $11(58 \%)$ were married and living with their spouse. Ten patients reported using a walking aid (either a single point stick or wheeled walker) at some point during their attendance at circuit program or at the six month review. Using a self-report measure, the median number of alls in the 6 months preceding attendance at the program was 6 (SE 6.8), compared to 2.0(SE 2.74) falls in the 6 months following completion of the classes, though this did not reach statistical significance. 
The mean number of years since the diagnosis of PD was made was 8 years (range 0-21). All patients had multiple co-morbidities, with the most common diagnoses being anxiety or depression, degenerative lumbar spine disease, osteoarthritis, osteoporosis and joint replacement surgery. No patients reported being active smokers, but $6(32 \%)$ were ex-smokers. The mean Body Mass Index was $23.8 \mathrm{~kg} /$ $\mathrm{m}^{2}$ (normal range 18.5-25). Ten patients had their cognition screened using a validated tool, either the Mini Mental Status Examination, ${ }^{31}$ Rowland Universal Dementia Assessment Scale ${ }^{32}$ or the Montreal Cognitive Assessment ${ }^{33}$ and scores ranged from 23-30 out of 30 .

Twelve of the patients (63\%) lived in low-set accommodation. Ten patients reported using a walking aid (either a single point stick or wheeled walker) at some point during their attendance at $\mathrm{CBC}$ or on review. Using a self-report measure, the median number of falls in the 6 months preceding attendance at $\mathrm{CBC}$ was 6(SE 6.8), compared to 2.0(SE 2.74) falls in the 6 months following completion of the classes, though this did not reach statistical significance. The Step Test is a test of dynamic balance that requires the subject to step on and off a block as quickly as possible in 15 seconds, whilst supported on the contra lateral leg. ${ }^{24}$ Performance on the Step Test improved significantly from baseline to completion with more steps taken within the allowed time. Using the right leg for support, on average the right step test the mean (SE) number of steps taken in 15 seconds improved from 9.33(0.72) to $11.16(0.87)$ following the intervention $(\mathrm{p}=0.001)$. The mean left step test mean (SE) number of steps taken when supported on the left leg improved from $9.55(0.65)$ to $11.34 \mathrm{~s}(0.58)(\mathrm{p}=0.005)$. There was also a statistically significant decline in performance in both left and right step tests at the 6 month review. The mean (SE) number of steps taken with left step support returned to the baseline level of 9.5(0.85) steps $(p=0.005)$. The other clinical indirect measures of balance (Timed Up and Go and- TUG, Functional Reach) whilst not clinically significant showed a similar trend of improved performance immediately after completion of the program $\mathrm{CBC}$, with regression at 6 month review. Performance on the Functional Reach test neared, but did not reach statistical significance $(\mathrm{p}=0.06)$ (Figure 1$)$.

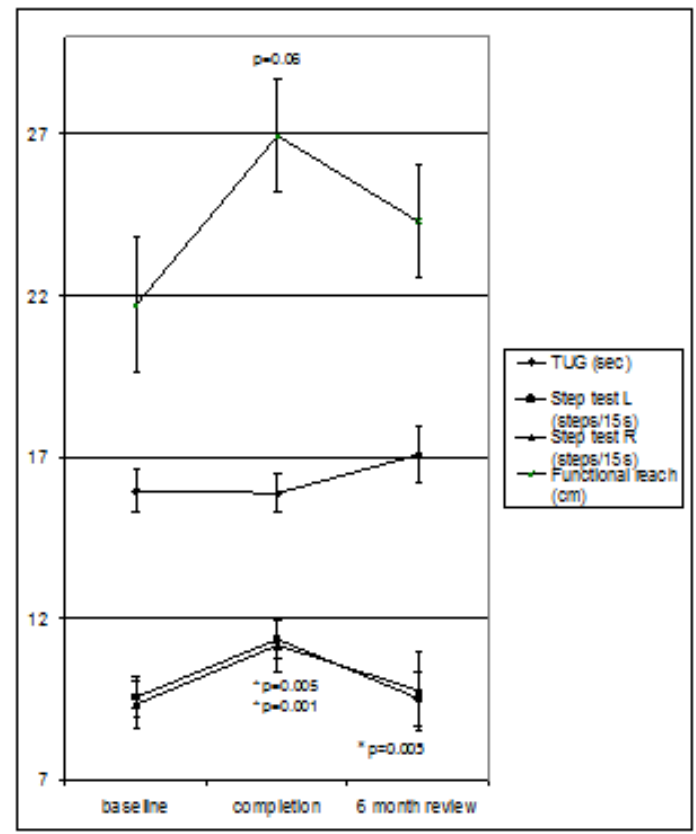

Figure I Balance measures.
Changes inThere were corresponding trends on the measures of walking performance, but again these did not reach statistical significance. On the Ten Meter Walk Test, mean (SE) velocity increased from 1.03(0.07) to 1.11(0.08) metres per second after completion of the program $\mathrm{CBC}$, but was not maintained at the 6 month follow-up $(0.99 \mathrm{~m} / \mathrm{s}$ [SE 0.08]) (Figure 2). The average distance walked by the subjects in the Six Minute Walk Test improved from 333.8 metres (SE 27.6) to 367.4 metres (SE 28.3) immediately after the intervention, but had declined to below baseline levels at 6 months $(316.9 \mathrm{~m}$ [SE 36.2]).

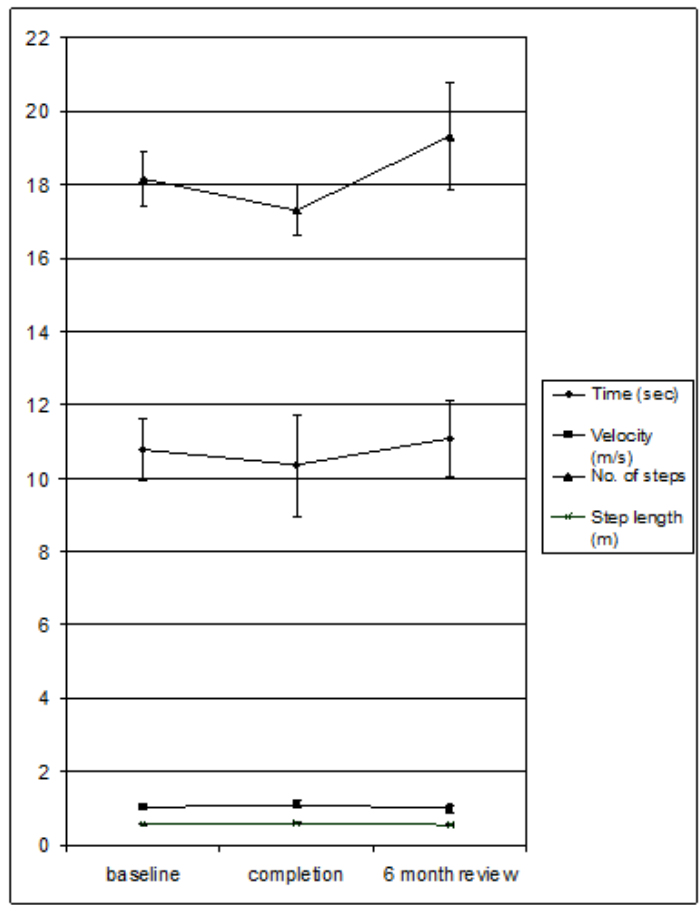

Figure 2 Walking performance measure-ten meter walk test.

Four patients attended two blocks of circuit program CBC during the study period. For these patients the mean duration of time between admissions was 20 months (range 11-32 months). All patients required the use of a walking aid by the time of their second attendance. For all outcome measures there was a tendency to improvement from re-admission to completion again, with subsequent regression at six months. Scores were generally worse than those from the first admission, comparing the individuals both to themselves and to the group means. The mean Six Minute Walk Test distance for this subset of patients on final review was $252.5 \mathrm{~m}$ (SE 17.5).

Eighteen patients completed the EQ-5D Questionnaire (one patient had died prior to the time of the survey mail out). The percentage of patients reporting any problems (either 'some' or 'severe') in the five domains are detailed in Figure 3. The majority of patients reported difficulties with mobility (89\%), usual activities $(83 \%)$, pain $(61 \%)$ and anxiety/depression $(56 \%)$ prior to undertaking the program $\mathrm{CBC}$. The number of patients reporting problems increased in all domains after completion of the program $\mathrm{CBC}$, with all patients $(100 \%)$ reporting difficulty with mobility at this time. However, no few patients rated their level of difficulty as 'severe'. Although the median EQ-5D utility score declined 6 months post the program CBC, however, the change was not statistically significant (from 0.582 
[SE 0.06] to 0.469 [SE 0.06] (Figure 4). The mean self-rated health status (0-100) was essentially unchanged after completion of circuit program CBC (from 53.97 [SE 3.76] to 55.35 [SE 4.41]) (Figure 5). The mean overall satisfaction score for the program (0-7) was 5.72 (SE 0.27). No adverse incidents were reported by the people attending the circuit training classes.

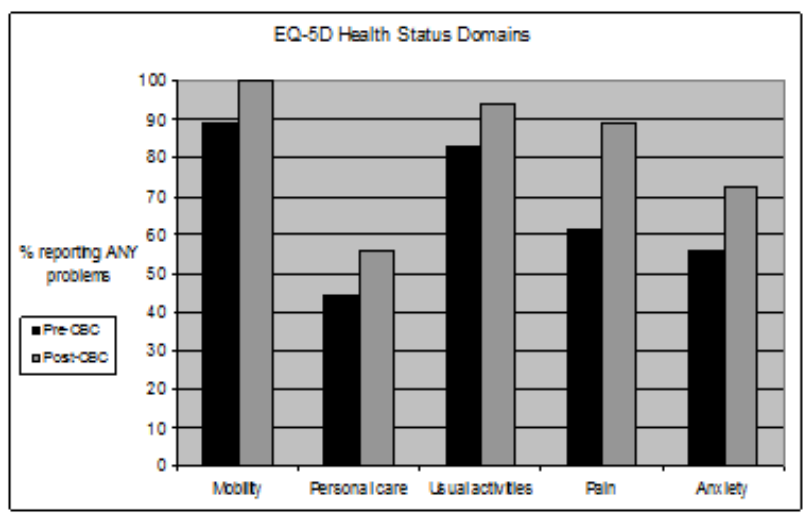

Figure 3 Self-reported health states.

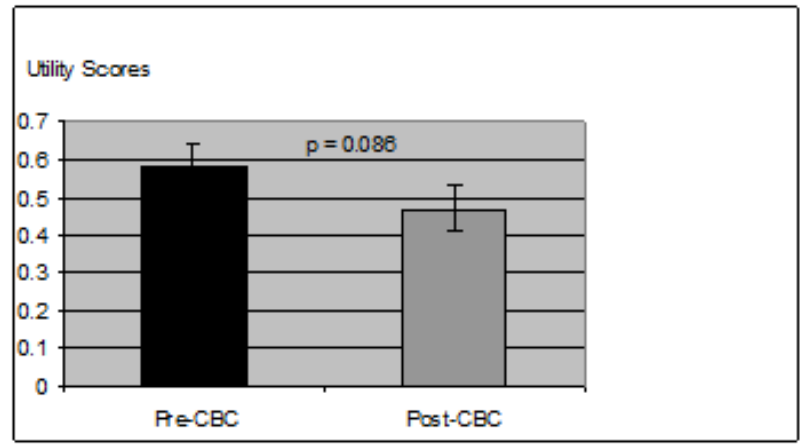

Figure 4 Quality of life- median EQ-5D utility score.

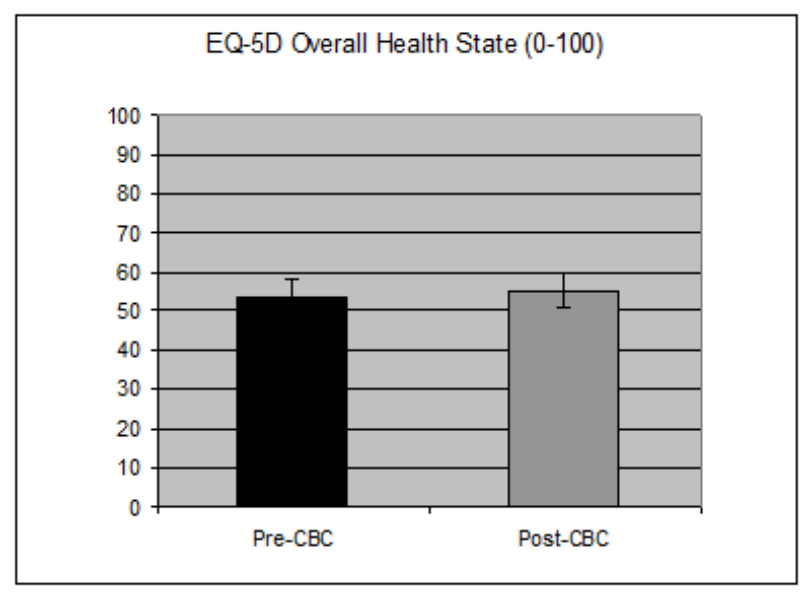

Figure 5 Quality of life -Visual analogue scale.

\section{Discussion}

This pilot study is the first to investigate the effects of circuit training program fora light to the experience of a sample of community-dwelling personsatients with PD who haveith balance or mobility impairment. Consistent with previous research, ${ }^{3,4,7}$ it confirms that they fall frequently. This program it has shown that attendance at $\mathrm{CBC}$ was effective in improving some aspects of dynamic balance in the target population, as measured by the Step Test. This improvement was not sustained at 6 months post-intervention. Whilst the physical gains were modest and relatively short-lived, it is important to consider that these may be very significant changes to the individual living with this degenerative disease.

There was a trend towards improvement following the intervention for the other clinical measures of balance and walking performance and a reduction in the self-reported number of falls, but they did not reach statistical significance. This lack of significant change may be due to the small sample size, with the study inadequately powered to demonstrate a significant change. Alternatively, the Step Test may be a more sensitive measure of balance than the other indirect measures of balance. This would be in-keeping with other precious research has which showed that for people in a group of patients with PD and no history of falls, the Step Test was the only test of self-induced imbalance that demonstrated a significant change after physical therapy. ${ }^{27}$ Despite these improvements after completion of CBC, the Step Test scores were still well below those of healthy elderly subjects. ${ }^{24,27}$

A demonstrated reduction in falls is would be the desired outcome of any balance such program. C However, consistent with the recent meta-analysis, ${ }^{22}$ in this study an improvement in balance did not translate into a significant reduction in fall frequency. One explanation may be could argue that there was no may not have been an improvement at 6 months. This decline may represent the progressive nature of PD. More frequent post discharge follow up may assist in monitoring the decline but equally there has not been a deterioration in relation to baseline that one would have expected with disease progression. It would be interesting to look at the distributions of the falls over a shorter period of time such as in the 3 months pre and post intervention.

The mean baseline Six Minute Walk Test distance of $333.8 \mathrm{~m}$ was lower than those published in other studies, which report distances of $392-546 \mathrm{~m}$ in patients with mild to moderate PD..$^{25,28,34}$ The patients in this study also walked more slowly in the Ten Meter Walk Test, where the average time taken to complete the test was between $10-11 \mathrm{sec}$, compared to $8.8 \mathrm{sec}$ previously reported reported in the study by Schenkman et al. ${ }^{28}$ This may be due to differences in disease severity, however this is difficult to assess, as data pertaining to disease stage was not available for analysis in the current study. Balance is one of many elements that may contribute to walking performance in $\mathrm{PD} .^{21}$ As the $\mathrm{CBC}$ circuit training program specifically at TPCH targeted multiples aspects of balance including lower limb strength and coordination. , It is possible that it may be the case that the included exercises did not provide sufficient enough actual walking practice to result in a significant change in the two measures of walking performance. The patients who attended the program twice may have performed more poorly due to disease progression over time, or may have had more severe motor impairment which led to re-referral. Patients in the current study attended once a week over the course of the program. A more intensive program may be required to result in significant changes in walking ability.

Patients in this study rated their QoL relatively poorly both before and after the circuit classes $\mathrm{CBC}$. On the overall measure of health 
status, the mean score of the survey respondents was at least $25 \%$ lower than UK population norms for a similar age range for the EQ$5 \mathrm{D} .^{35}$ This is consistent with the severe and wide-ranging effects of PD symptoms and the progressive nature of the disease. Participation in this program $\mathrm{CBC}$ did not appear to negate these effects. The high number of people frequency reporting of mobility impairment, activity limitation and anxiety/depression I was notable, and consistent with the known motor and neuropsychiatric sequelae of PD. However, the frequency of reported problems with pain was significant, and warrants further investigation.

Within the circuit class model a staff to patient ratio of $1: 5$ is supported. As a result it has been suggested that circuit class training may be a more efficient means of providing physiotherapy interventions when compared to more traditional one to one models. ${ }^{16}$ Future research is required to further investigate the cost benefit of such a model for people with PD. Patient satisfaction with the program was remained high, which suggesting that there may be other potential benefits to participation. This may include socialization, education and improved identification of needs and access to other services. ${ }^{18}$ These benefits may themselves be enough to rationalize the cost of providing such a service.

This pilot study has a number of limitations. The relatively small, non-randomised sample and absence of a control group restricts the generalizability of the results. The retrospective study design meant it was not possible to control for stage of disease, co-morbidities or the timing of testing with medication dosages, all of which have the potential to affect performance. However, research has shown that performance on balance and mobility assessments does not change significantly across the medication cycle in patients with PD ${ }^{36}$ Asking patients to recall their QoL is likely to be less accurate than surveying them at the time, particularly in a patient population who may be experiencing cognitive decline. Despite this, the QoL data obtained provides a unique insight into the daily experience of the sample population. This study raises the question that whether subgroups with cognitive impairment respond better or worse to the intervention.

\section{Conclusion}

For this group of patients, circuit balance classes were a safe and effective means of providing balance and mobility rehabilitation. This study has shown that circuit balance classes result in a shortterm improvement in the balance of community-dwelling patients with PD who have balance or mobility impairment. The improved performance was not sustained at 6 months following the intervention. While self-rated QoL wais poor and appeared to be unaffected by the intervention, but overall satisfaction with the program was high. FIt is recommended that further research be undertaken required to confirm the preliminary findings of this pilot study, in the form of a prospective randomised controlled trial. Even a modest benefit may be enough to justify provision of circuit balance classes, given the high burden of disease and cost of managing the complications of PD, but a more formal cost-effectiveness analysis would allow such conclusions to be drawn.

This research may assist in the education of patients with PD in ambulatory rehabilitation to have realistic expectations of the possible outcomes of attendance at $\mathrm{CBC}$. It may also assist those working to evaluate the efficiency of program and to develop rehabilitation programs for patients with PD to more effectively tailor the content and frequency of their programs.

\section{Acknowledgements}

We would like to thank the patients and the staff of the Rehabilitation Day Therapy Unit at the Prince Charles Hospital in Queensland.

\section{Conflict of interest}

The author declares no conflict of interest.

\section{References}

1. Whetten-Goldstein K, Sloan F, Kulas E, et al. The burden of Parkinson's disease on society, family, and the individual. $J$ Am Geriatr Soc. 1997;45(7):844-849.

2. Living with parkinson's disease. Challenges and positive steps for the future. Australia: Access Economics Pty Limited; 2007.

3. Koller WC, Glatt S, Vetere-Overfield B, et al. Falls and Parkinson's disease. Clin Neuropharmacol. 1989;12(2):98-105.

4. Boonstra TA, van der Kooij H, Munneke M, et al. Gait disorders and balance disturbances in Parkinson's disease: clinical update and pathophysiology. Curr Opin Neurol. 2008;21(4):461-471.

5. Pasco JA, Sanders KM, Hoekstra FM, et al. The human cost of fracture. Osteoporos Int. 2005;16(12):2046-2052.

6. Haleem S, Lutchman L, Mayahi R, et al. Mortality following hip fracture: trends and geographical variations over the last 40 years. Injury. 2008;39(10):1157-1163.

7. Temlett JA, Thompson PD. Reasons for admission to hospital for Parkinson's disease. Intern Med J. 2006;36(8):524-526.

8. Hely M, Morris J, Traficante R, et al. The Sydney multicentre study of Parkinson's disease: progression and mortality at 10 years. J Neurol Neurosurg Psychiatry. 1999;67(3):300-307.

9. Sidaway B, Anderson J, Danielson G, et al. Effects of long-term gait training using visual cues in an individual with Parkinson disease. Phys Ther. 2006;86(2):186-194.

10. Franchignoni F, Martignoni E, Ferriero G, et al. Balance and fear of falling in parkinson's disease. Parkinsonism Relat Disord. 2005;11(7):427-433.

11. Kamata N, Matsuo Y, Yoneda T, et al. Overestimation of stability leads to a high frequency of falls in patients with Parkinson's disease. Clin Rehabil. 2007;21(4):357-361.

12. Deane K, Jones DE, Playford ED, et al. Physiotherapy versus placebo or no intervention in Parkinson's disease. Cochrane Database of Syst Rev. 2001;7:CD002817.

13. Howe TE, Rochester L, Jackson A, et al. Exercise for improving balance in older people. Cochrane Database of Syst Rev. 2007;(11):CD004963.

14. Faherty CJ, Shepherd KR, Herasimtschuk A, et al. Environmental enrichment in adulthood eliminates neuronal death in experimental Parkinsonism. Brain Res Mol Brain Res. 2005;134(1):170-179.

15. Sutoo D, Akiyama K. Regulation of brain function by exercise. Neurobiology of Disease. 2003;13(1):1-14.

16. Bulat T, Hart-Hughes S, Ahmed S, et al. Effect of a group-based exercise program on balance in elderly. Clin Interv Aging. 2007;2(4):655-660.

17. Suchowersky O, Gronseth G, Perlmutter J, et al. Practice parameter: neuroprotective strategies and alternate therapies for Parkinson disease (an evidence-based review). Report of the quality standards subcommittee of the american academy of neurology. Neurol. 2006;66(7):976-982.

18. Gage H, Kaye J, Owen C, et al. Evaluating rehabilitation using costconsequences analysis: an example in Parkinson's disease. Clin Rehabil. 2006;20(3):232-238 
19. van de Port IGL, Wevers L, Roelse H, et al. Cost-effectiveness of a structured progressive task-oriented circuit class training programme to enhance walking competency after stroke: The protocol of the FITStroke trial. BMC Neurology. 2006;9:43.

20. Deane K, Jones DE, Ellis-Hill C, et al. Physiotherapy for Parkinson's disease: a comparison of techniques. Cochrane Database of Syst Rev. 2001;6:CD002815.

21. Keus SHJ, Bloem BR, Hendriks EJM, et al. Evidence-based analysis of physical therapy in Parkinson's disease with recommendations for practice and research. Mov Disord. 2007;22(4):451-460.

22. Goodwin VA, Richards SH, Taylor RS, et al. The effectiveness of exercise interventions for people with Parkinson's disease: A systematic review and meta-analysis. Mov Disord. 2008;23(5):631-640.

23. http://guidance.nice.org.uk/CG35

24. Hill KD, Bernhardt J, McGann AM, et al. A new test of dynamic standing balance for stroke patients: reliability, validity and comparison with healthy elderly. Physiotherapy Canada. 1996;48(4):257-262.

25. Falvo MJ, Earhart GM. Six-minute walk distance in persons with Parkinson disease: A heirarchical regression model. Arch Phys Med Rehabil. 2009;90(6):1004-1008.

26. Rossier P, Wade DT. Validity and reliability comparison of 4 mobility measures in patients presenting with neurologic impairment. Arch Phys Med Rehabil. 2001;82(1):9-13.

27. Stankovic I. The effect of physical therapy on balance of patients with Parkinson's disease. International Journal of Rehabilitation Research. 2004;27(1):53-57.
28. Schenkman M, Cutson TM, Kuchibhatla M, et al. Reliability of impairment and physical performance measures for persons with Parkinson's disease. Phys Ther. 1997;77(1):19-27.

29. Johnson JA, Coons SJ, Ergo A, et al. Valuation of EuroQOL (EQ5D) health states in an adult US sample. Pharmacoeconomics. 1998;13(4):421-433.

30. Robin R, de Charro F. EQ-5D: a measure of health status from the euroqol group. Ann med. 2001;33(5):337-343.

31. Folstein M, Folstein S, McHugh P. Mini-mental state:A practical method for grading the mental state of patients for the clinician. J Psychiatr Res. 1975;12(3):189-198.

32. Storey JE, Rowland JTJ, Conforti DA, et al. The rowland universal dementia assessment scale (RUDAS): a multicultural cognitive assessment scale. Int Psychogeriatr. 2004;16(1):13-31.

33. Nasreddine ZS, Phillips NA, Bedirian V, et al. The montreal cognitive assessment (MOCA): a brief screening tool for mild cognitive impairment. J Am Geriatr Soc. 2005;53(4):695-699.

34. Canning CG, Ada L, Johnson JJ, et al. Walking capacity in mild to moderate Parkinson's Disease. Arch Phys Med Rehabil. 2006;87(3):371375.

35. Kind P, Hardman G, Macran S. UK Population norms for EQ-5D. The university of York centre for health economics, 1999. Discussion paper 172. 1999. p. $1-98$.

36. Campbell F, Ashburn A, Thomas P, et al. An exploratory study of the consistency of balance control and the mobility of people with Parkinson's disease (PD) between medication doses. Clin Rehabil. 2003;17(3):318-324. 